The BDI and CESD performed better, however, when their cutoff points for a diagnosis of depression were raised beyond those validated in the general population-supporting the notion that self-report scales record additional somatic depressive symptoms in hemodialysis patients that are not true indicators of depression. In further analyses, the CESD was found to be more accurate than the BDI at identifying individuals with depression.

The authors conclude that at the proposed new cutoffs the BDI and CESD are sufficiently accurate to be used as screening tools; they suggest that these tools be used to evaluate dialysis patients for depression at approximately yearly intervals, with patients with positive results being formally assessed by a physician or referred for psychiatric assessment. They do not recommend the CDI for screening because of its low sensitivity.

Original article Hedayati SS et al. (2006) The predictive value of self-report scales compared with physician diagnosis of depression in hemodialysis patients. Kidney Int 69: 1662-1668

\section{Ethnic differences in risk of MI among patients with ESRD}

Among patients with chronic renal insufficiency, cardiovascular disease (CVD) risk is higher in racial and ethnic minorities than in whites. Young et al. have investigated whether there are ethnic differences in incident myocardial infarction (MI) rates among patients with end-stage renal disease (ESRD).

The analysis included 271,102 patients who initiated renal replacement therapy in the US between January 1995 and December 2000, and were eligible for Medicare insurance coverage. Prevalent CVD was present in $43.8 \%$ of patients. Overall, $52.1 \%$ of participants were white, $31.6 \%$ were African American, $3.0 \%$ were Asian American and $11.5 \%$ were Hispanic.

Prevalent CVD was most common in whites (60.0\% of all CVD cases), followed by African Americans (25.6\%), Hispanics (10.1\%), Asian Americans $(2.57 \%)$ and other ethnicities (1.69\%). Unadjusted MI event rates were approximately twice as high in patients with prevalent CVD than in those without CVD. Factors associated with higher nonfatal and combined (i.e. fatal and nonfatal) MI rates among patients with and without CVD were white race, increasing age, and inability to walk. Among patients with prevalent CVD, whites had the highest risk of combined $\mathrm{Ml}$; risk was $35 \%$ lower in African Americans, 26\% lower in Asian Americans, and 28\% lower in Hispanics, after adjustment for confounding factors. Whites also showed the greatest risk of combined MI among patients without prevalent CVD.

The authors conclude that incident MI risk is higher in ESRD patients with prevalent CVD than in those without, and that MI risk among ESRD patients is higher in whites than in other ethnic groups.

Original article Young BA et al. (2006) Racial and ethnic differences in incident myocardial infarction in end-stage renal disease patients: the USRDS. Kidney Int 69: 1691-1698

\section{A predictive index of disease progression in IgA nephropathy}

Immunosuppressive therapy is effective against IgA nephropathy, but is not always suitable because of serious adverse effects. Magistroni et al. retrospectively reviewed 237 IgA nephropathy cases diagnosed from 1981 to 2004 to identify indicators of disease progression that might help to guide treatment.

Multivariate analysis of clinical and pathologic data revealed serum creatinine level, urinary protein excretion, mean arterial blood pressure, and age at biopsy to be the main risk factors for development of end-stage renal disease. A clinical predictive index (CPI) was calculated from these factors, assigning 2 points for serum creatinine level $>1.4 \mathrm{mg} / \mathrm{dl}$ ( $>124 \mu \mathrm{mol} / \mathrm{l}), 1$ point for proteinuria $>1 \mathrm{~g} /$ day, 1 point for hypertension and 1 point for age $>30$ years at biopsy. Patients with a CPI score of 0-2 (low CPI score) had a 10-year renal survival rate of $91.7 \%$, compared with $35.0 \%$ for patients with a CPI score of 3-5 (high CPI score; $P<0.001)$. Validation of the model in a group of 73 patients from another treatment center produced similar results. Histological grading by Lee classification and proteinuria $>1 \mathrm{~g}$ /day were shown to be independent predictors for doubling of serum creatinine level in the group with low CPI scores.

Although the study did not demonstrate a clear benefit for immunosuppressive therapy, the authors recommend this treatment for patients with a high CPI score and those with a low $\mathrm{CPI}$ score who also have proteinuria $>1 \mathrm{~g} /$ day 